\title{
Minimization of Intra-Operative Shaping of Orthopaedic Fixation Plates: A Population-Based Design
}

\author{
Habib Bou-Sleiman ${ }^{1, \star}$, Lucas E. Ritacco ${ }^{2}$, Lutz-Peter Nolte ${ }^{1}$, \\ and Mauricio Reyes ${ }^{1}$ \\ 1 Institute for Surgical Technology and Biomechanics, \\ University of Bern, Stauffacherstrasse 78, 3014 Bern, Switzerland \\ \{habib.bousleiman, mauricio.reyes\}@istb.unibe.ch \\ 2 Hospital Italiano de Buenos Aires, Gascón 450, Buenos Aires, Argentina
}

\begin{abstract}
In this paper we present a new population-based method for the design of bone fixation plates. Standard pre-contoured plates are designed based on the mean shape of a certain population. We propose a computational process to design implants while reducing the amount of required intra-operative shaping, thus reducing the mechanical stresses applied to the plate. A bending and torsion model was used to measure and minimize the necessary intra-operative deformation. The method was applied and validated on a population of 200 femurs that was further augmented with a statistical shape model. The obtained results showed substantial reduction in the bending and torsion needed to shape the new design into any bone in the population when compared to the standard mean-based plates.
\end{abstract}

Keywords: Orthopaedic implant design, population-based analysis, bone fixation plate.

\section{Introduction}

Bone fixation plates are commonly used in orthopaedic surgeries to preserve, maintain, and help restore the original anatomy of a diseased or fractured bone. The success of reconstructive and corrective interventions heavily relies on the proper design and application of the implants. Current trends tend to offer fixation plates that are pre-contoured to the specific target location in which they are supposed to function 12 . Pre-contouring is commonly based on the average anatomy of the target population, or on a template bone considered as a representative of that population. To date, the available pre-contoured plates are not capable of providing an optimal fit to all operated patients. This is mainly due to the differences in morphology of the human skeleton between and within different populations. Factors such as age, gender, and ethnic origin play an important role in defining the morphology of the bones [3].

\footnotetext{
^ Corresponding author.
} 
Furthermore, fixation plates are manually shaped during the surgery in order to adapt them to the patient-specific anatomy, a delicate and time-consuming procedure that is prone to high inaccuracies 4 . Such procedures require wide exposure of the bone [2] and longer surgical time, thus increasing the risks and costs of the intervention. The development of locked internal fixators and anatomically pre-shaped plates reduced the need of plate bending [1 2 56 6], however, accurate patient-specific implant pre-contouring is still not accessible.

In [7], the authors propose a method to design plates by searching through the parametric space of a statistical shape model of the bones. They search for the most significant population-specific shape variability patterns that affect the fit of the fixation plate using criteria based on surface-to-surface distances. They finally propose manual changes to the current design and prove that the new plate fits more bones from the population according to the same design criteria.

In this paper, we present a new population-based automatic approach to the design of orthopaedic fixation plates. We propose a design that minimizes the maximum amount of intra-operative manual bending and torsion that are to be applied to the plate. The proposed deformation model and metrics go in agreement with the type of deformation the surgeon applies during the intervention. The design criteria are more clinically significant than those used in [7. The benefit of such method is two-fold. Explicitly, less mechanical stresses are applied to the plate and therefore a better long-term mechanical stability is expected. Whereas the lesser required shaping implicitly indicates a better pre-operative surface-to-surface fit.

\section{Methods}

\subsection{Experimental Data}

A population of 200 segmented computed tomography (CT) datasets with varying image parameters and scanners was used in this work. Point distribution models (PDM) of the left femur were generated and aligned. Dense point-topoint correspondence was computed using an image-based log-domain demons registration with a polyaffine regularization [8].

Two patches of interest have been selected, outlined, and extracted from the initial datasets by an experienced orthopaedic surgeon. The patches are located on the distal medial and the distal lateral sections of the femur. Fig. 1a-b shows these two patches.

Using the pre-established point-to-point correspondence, the vertices of the chosen patches were propagated throughout the PDM in order to obtain 200 samples of each type of patch that are subsequently realigned. In the present case, each distal medial patch is composed of 1,343 surface points whereas its lateral counterpart is composed of 1,625 points. These patches are considered as both bone surfaces and potential new designs of the contact surface of the fixation plate. The two terms plates and patches will be used interchangeably throughout the rest of this paper. 


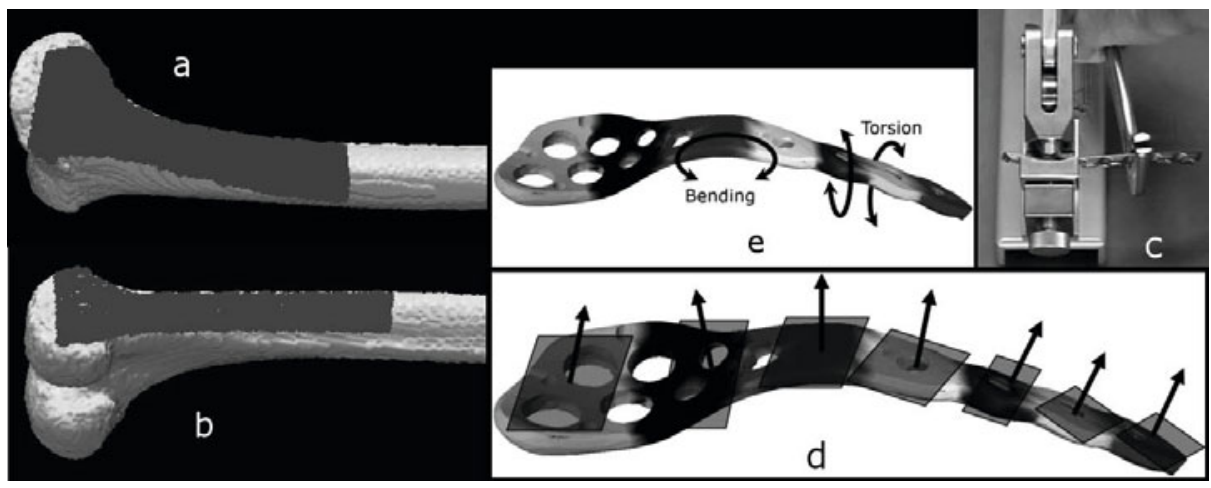

Fig. 1. Selection of the $(a)$ medial and (b) lateral distal patches of the femur. (c) Intraoperative bending of a fixation plate. $(d)$ Example of how the plate can be divided into discrete sections. A plane is fitted to each individual section and represented by its normal vector. (e) Illustration showing the directions of bending and torsion.

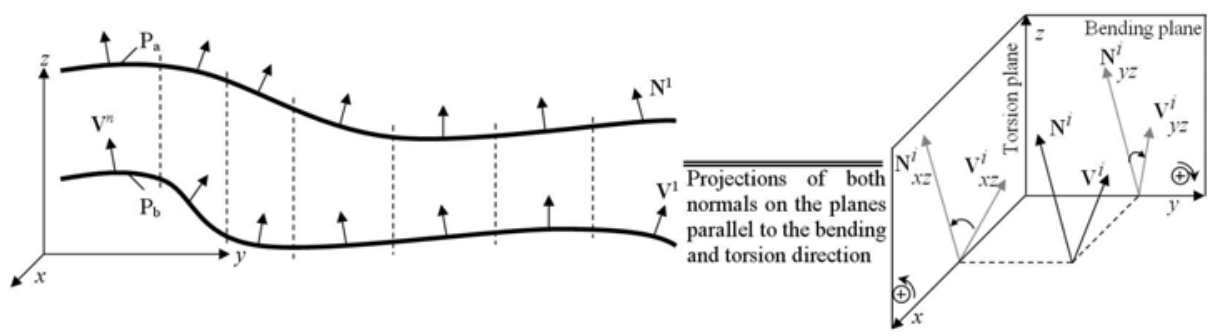

Fig. 2. Illustration of how the method computes the bending and torsion angles required to shape one patch $\left(\mathrm{P}_{a}\right)$ into another $\left(\mathrm{P}_{b}\right)$. Both components are measured relative to the main axis of the patches, assuming the main axis is aligned with the $y$-axis of the coordinate system. In this example, the bending component is the angle between the projections of the normals onto the yz-plane, whereas the torsion component is the angle between the projections of the normals onto the xz-plane.

\subsection{Plate Discretization and Representation}

We propose to first represent the contact surface of a fixation plate (or the bone patch) by dividing it into discrete sections lengthwise and fitting a plane to each section. The number of sections depends on the size of the implant and anatomical location. It should be small enough to capture the anatomical features, but large enough not to be affected by local noise in the surface. The size of the individual sections should also be within the operational limits of the common shaping tools (see Fig. 1, ). Each plane is then represented by a vector normal to it. An example of how the plate can be divided is shown in Fig. 11. 


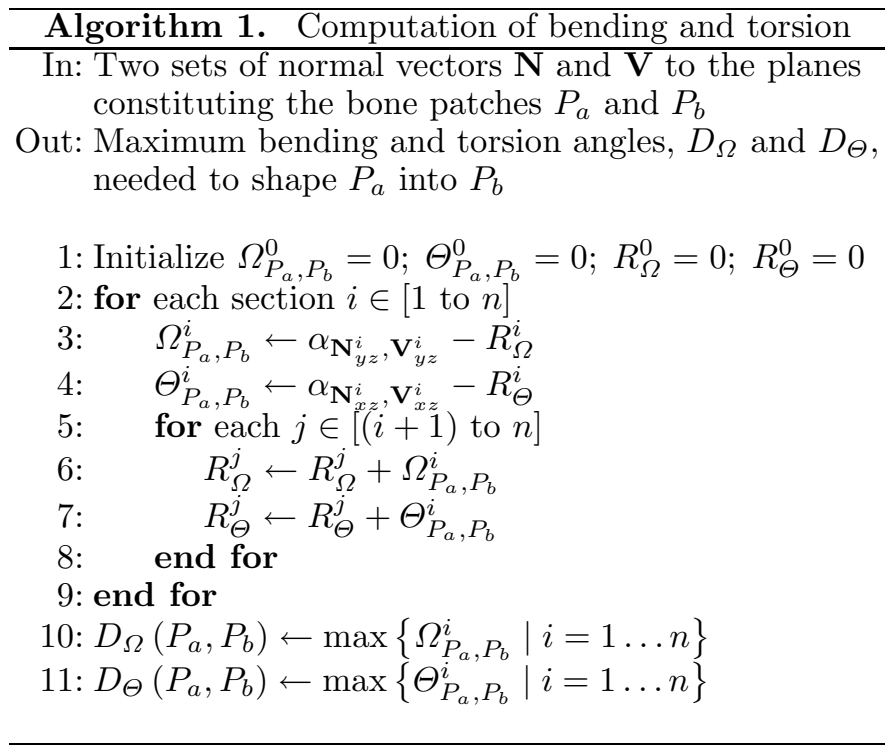

\subsection{Measurement of Bending and Torsion}

The key formulation of our solution is to examine the effect of shaping each section of the implant on the rest of the sections and compute the amount of deformation needed to shape one patch to another based on currently available shaping tools. The angles between two corresponding normals to the planes are split into two independent components, namely bending and torsion, both relative to the main axis of the patch. Fig. 2 illustrates how the patches are discretely represented by a set of normal vectors and how the bending and torsion angles are measured.

The method used to measure how much bending $\left(D_{\Omega}\right)$ and torsion $\left(D_{\Theta}\right)$ are required to shape one patch into another is formulated in Algorithm 1, where $n$ is the number of sections per patch, $\alpha$ is an angle between two vectors, $\Omega$ and $\Theta$ are the vectors of bending and torsion angles, and $R_{\Omega}$ and $R_{\Theta}$ are the vectors of residual bending and torsion angles, both respectively. Letting $P_{a}$ be the patch to be contoured to $P_{b}$, the algorithm starts from one end of the patches, say the proximal end, and sequentially goes along the main axis until the other end is reached. This process is analogous to drum pressing the plate against a template until it acquires its shape. It also mimics what the surgeon would do during the intervention. It starts by initializing the angles to zero, indicating an alignment of the first normals without any deformation of the patch or plate (line 1). The algorithm then proceeds by iterating over two main steps. The first computes the bending and torsion required to align two corresponding normal vectors while taking into account residual angles from previous steps (lines 3-4). The second is an update step that computes the vector of residual angles and 
consecutively assigns its elements to the remaining normal vectors in the chain of sections along the patch (lines 6-7). This update step describes the effect of applying a local deformation on the rest of the plate. Our interest is to compute the maximum needed local deformation. Thus the highest values in the lists of bending and torsion are extracted and stored for later processing (lines 10-11). These values are to be later minimized since they are the maximal forces that a plate design will undergo during the surgery.

\subsection{Finding the Optimal Template from a Population}

By applying the analysis described above to the whole population, one is able to measure pair-wise maximal bending and torsion angles needed to shape any one patch into another. Our goal is to find the patch that simultaneously minimizes both components among the studied population. Consistent with the notation presented in Algorithm 1, we propose to optimize our metrics using Eq. (1) through Eq. (3). In Eq. (3), NewDesign is the index of the patch that requires the least amount of physical shaping, and $p$ the size of the population. The weighting factors $\omega_{\Omega}$ and $\omega_{\Theta}$ are used to impose optional non-equal significances on both components. In our experiments, we used unity weights indicating equal contribution of bending and torsion to the design criterion.

$$
\begin{aligned}
d_{\Omega}^{a} & =\max \left\{D_{\Omega}\left(P_{a}, P_{b}\right) \mid b=1 \ldots p\right\} ; \\
d_{\Theta}^{a} & =\max \left\{D_{\Theta}\left(P_{a}, P_{b}\right) \mid b=1 \ldots p\right\} ; \\
\text { NewDesign } & =\underset{a}{\operatorname{argmin}}\left\{\sqrt{\omega_{\Omega} d_{\Omega}^{a^{2}}+\omega_{\Theta} d_{\Theta}^{a}} \mid a=1 \ldots p\right\} .
\end{aligned}
$$

\subsection{Further Optimization}

In order to avoid falling into local minima and to ensure that the method finds the optimal plate design, we further examined the three best ranked patches from the previous steps. We generated a statistical shape model [9] of the initial populations and retained the first 42 principal components capturing $99 \%$ of the natural shape variability. The parameters of the three patches within the statistical space were recovered using a least-squares approximation $\mathbf{c}=\mathbf{Q}^{+} \mathbf{y}$, where $\mathbf{c}$ is the vector of shape parameters, $\mathbf{Q}$ the matrix of eigenvectors scaled by the corresponding eigenvalues, and $\mathbf{y}$ the vector of coordinates of the surface points. Latin hypercube sampling [10] was used to generate 100 instances in the statistical vicinity of each of the best choices. Latin hypercube sampling was preferred over uniform or random dense sampling since it offers a similar space coverage with a substantially lower number of samples. The same search described earlier was applied again to the augmented populations.

\subsection{Finding an Adequate Number of Sections}

The number of sections per plate or bone patch is a fundamental parameter in the design process. Care must be taken while defining this parameter since it directly 

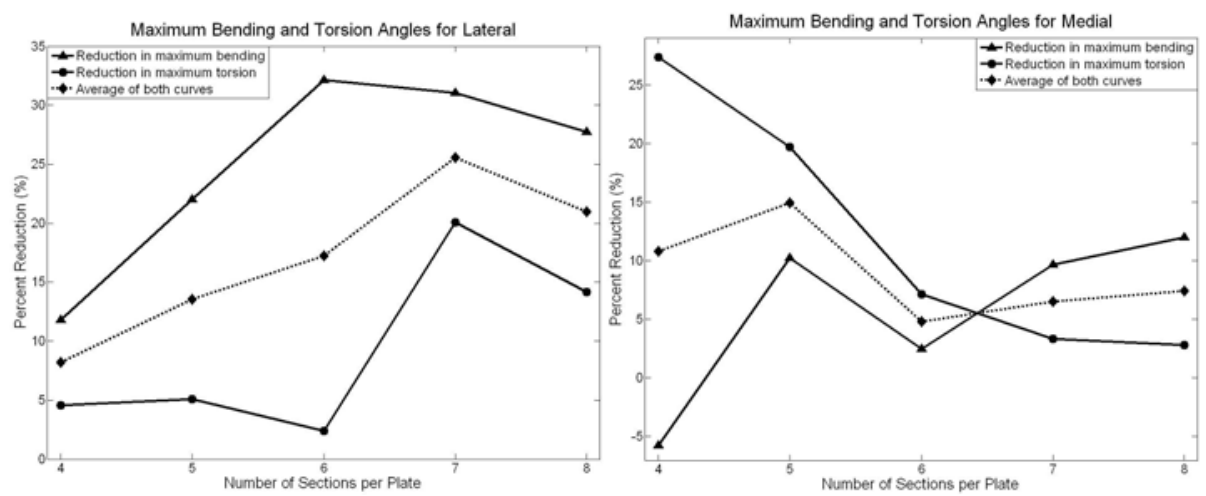

Fig. 3. Reduction in the maximum bending and torsion angles needed to shape the proposed design to any instance in the population, for lateral (left) and medial (right) plates. The reduction is relative to the results obtained for the standard design and plotted against the number of sections used to divide the bone patches or plates. The average curve has its peak at seven sections for the lateral case, whereas five sections resulted in a higher improvement for the medial dataset.

affects the quality of the final design. For each one of the considered anatomical sites, we tested the algorithm above using a varying number of sections per plate. We recorded the amount of improvement 11 over the standard mean-based designs. Ideally, the goal is to pick the number that yields the highest improvement in both bending and torsion components. We used the average values of both components as a selection criterion.

\section{$3 \quad$ Results}

The method described above was separately applied to the sets of bone patches extracted from the medial and lateral distal femur. The first step was to identify the optimal number of sections that divide each instance of the population. Fig. 3 shows the results of the corresponding tests, where seven and five dividing sections yielded the highest improvement for the lateral and medial cases, respectively.

Following the initial step, the configuration of the discretization pattern that yielded the best improvement was retained and applied to the whole population as well as to the standard design. Fig. 4 plots the obtained results and highlights the difference between the maximum required deformation for the standard plates and that for the enhanced designs. Table 1 shows a comparison between the standard plates and the proposed improved design. It also compares the highest pre-operative mean surface distances (MSD) measured for every case. The results indicate that the intra-operative deformation required

${ }^{1}$ Improvement is measured as the percent reduction in maximum bending and torsion from the values recorded for the standard design. 

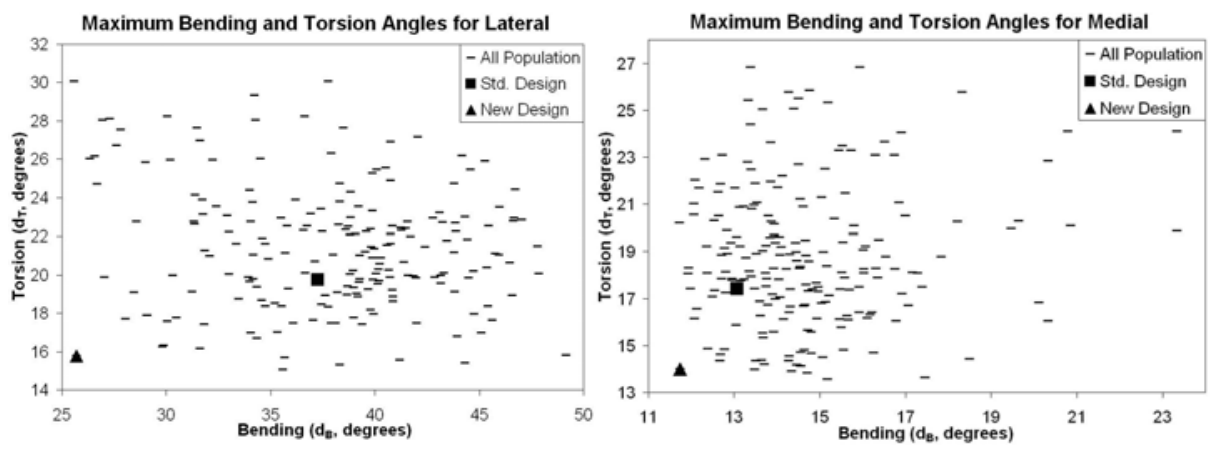

Fig. 4. Maximum bending and torsion required to shape any instance of the population (-) to all other instances, for lateral (left) and medial (right) plates. The new design

$(\boldsymbol{\Delta})$ resulted in lower bending and torsion angles than the standard design (ם).

to fit the standard plate to any potential patient exceeds that required by the new design. Consequently, this implies that it is easier to contour the new plate design to the patient-specific anatomy.

\section{Discussion}

In this paper we presented a novel population-based method to design precontoured orthopaedic fixation plates. The method minimizes the maximum deformation (bending and torsion) required to fit an implant to the patientspecific anatomy. The presented deformation model is consistent with the actual intra-operative deformation and complies with the current shaping tools. The results indicate that the population mean is not the optimal design, neither the MSD is the optimal design metric. The highest possible pre-operative MSD (preceding intra-operative shaping) between the plate and any bone surface is also reduced (medial) or almost unchanged (lateral) with the new design. The threedimensional surface representation of the bone patch designated as NewDesign can be used as a template to manufacture better pre-contoured implants.

We intend to apply the method presented herein to populations of different anatomical sites. Different population characteristics can also be included in the analysis such as gender, race, age group, etc. This would divide the population

Table 1. Comparison between the standard mean-based design and the new design proposed in this paper. The values refer to the highest measured angles and distances.

\begin{tabular}{r|ccc|ccc} 
& \multicolumn{3}{|c}{ Lateral Plate } & \multicolumn{3}{c}{ Medial Plate } \\
& Bending Torsion & MSD & Bending Torsion & MSD \\
\hline Standard Design & $37.25^{\circ}$ & $19.74^{\circ}$ & $23.15 \mathrm{~mm}$ & $13.07^{\circ}$ & $17.41^{\circ}$ & $17.03 \mathrm{~mm}$ \\
New Design & $25.27^{\circ}$ & $14.00^{\circ}$ & $23.22 \mathrm{~mm}$ & $11.74^{\circ}$ & $13.22^{\circ}$ & $14.36 \mathrm{~mm}$ \\
Improvement & $\mathbf{3 2 . 1 6 \%}$ & $\mathbf{2 9 . 0 8 \%}$ & $\mathbf{- 0 . 3 0 \%}$ & $\mathbf{1 0 . 1 8 \%}$ & $\mathbf{2 4 . 0 7 \%}$ & $\mathbf{1 5 . 6 8 \%}$
\end{tabular}


into subgroups that can be used to design population-specific implants. The population could also be divided using clustering methods based on the presented design metrics (and possibly others) to generate multiple more specific designs. We also propose to further extend this method and develop a simple guidance system that can be integrated into the manufactured implants. It should indicate the different sections and the way and amount by which the surgeon must deform the plate. An image-guided system that does not require the development of sophisticated shaping tools is an ultimate goal that is brought within reach by the methods and representation presented in this paper. It would allow for a smooth transfer from the design workbench to the operating theater. We also plan to carry out mechanical tests to assess the weighted contribution of bending and torsion to the design criteria and the impact of screw holes deformation.

Acknowledgments. This work was carried out within the frame of the National Center of Competence in Research, Computer-Aided and Image-Guided Medical Interventions (NCCR Co-Me), supported by the funds of the Swiss National Science Foundation (SNSF).

\section{References}

1. Perren, S.M.: Evolution and rationale of locked internal fixator technology. Introductory remarks. Injury 32(suppl.2), B3-B9 (2001)

2. Wagner, M.: General principles for the clinical use of the LCP. Injury 34(suppl. 2), B31-B42 (2003)

3. Schmutz, B., Wullschleger, M.E., Kim, H., Noser, H., Schutz, M.A.: Fit assessment of anatomic plates for the distal medial tibia. J. Orthop. Trauma 22, 258-263 (2008)

4. Frankle, M.A., Cordey, J., Frankle, M.D., Baumgart, F., Perren, S.: A retrospective analysis of plate contouring in the tibia using the conventional 4.5 (narrow) dynamic compression plate. J. Orthop. Trauma 8, 59-63 (1994)

5. Goyal, K.S., Skalak, A.S., Marcus, R.E., Vallier, H.A., Cooperman, D.R.: Analysis of anatomic periarticular tibial plate fit on normal adults. Clin. Orthop. Relat. Res. 461, 245-257 (2007)

6. Taljanovic, M.S., Jones, M.D., Ruth, J.T., Benjamin, J.B., Sheppard, J.E., Hunter, T.B.: Fracture fixation. Radiographics 23, 1569-1590 (2003)

7. Kozic, N., Weber, S., Büchler, P., Lutz, C., Reimers, N., Ballester, M.A.G., Reyes, M.: Optimisation of orthopaedic implant design using statistical shape space analysis based on level sets. Med. Image Anal. 14, 265-275 (2010)

8. Seiler, C., Pennex, X., Ritacco, L., Reyes, M.: Femur specific polyaffine model to regularize the log-domain demons registration. In: SPIE Med. Imaging. Florida (2011)

9. Davies, R., Twining, C.J., Taylor, C.J.: Statistical models of shape: optimisation and evaluation. Springer, London (2008)

10. McKay, M.D., Beckman, R.J., Conover, W.J.: A comparison of three methods for selecting values of input variables in the analysis of output from a computer code. Technometrics 21, 239-245 (1979) 